\title{
LEDA LLRF Control System Performance: Model and Operational Experience*
}

\author{
A.H. REGAN, S.I. KWON, Y.M. WANG, Los Alamos National Laboratory, Los Alamos, NM \\ C.D. ZIOMEK, ZTEC-Inc, Albuquerque, NM
}

\begin{abstract}
In modern industrial control, a relay experiment can be used for auto-tuning a PI controller. It gives the ultimate frequency and ultimate gain of the open loop system. Based on the ultimate gain and the ultimate frequency, the PI control parameters, the proportional gain, and the integration time, are determined. A relay experiment can be used to determine the sensitivity of a closed loop system against external disturbances. This paper addresses a method to estimate the sensitivity represented by the gain margin of a closed loop low-level RF (LLRF) control system based on the Matlab/Simulink model of the Low Energy Demonstration Accelerator (LEDA) RFQ. This paper will discuss some of the modeling performed and our operational experience to date.
\end{abstract}

\section{INTRODUCTION}

This paper discusses a method to measure the amplitude margin of the LLRF control system. The method is based on a relay experiment. Basically a relay experiment uses a square wave as the disturbance input, rather than a sine wave. Because the Fourier transform of a square wave contains a multitude of frequencies, rather than a single one, it is a more efficient way of evaluating the performance of the system. It gives the ultimate frequency and ultimate gain of the open loop system. Based on the ultimate gain and the ultimate frequency, PID controller parameters, i.e., the proportional gain $K_{P}$, the integration time $T_{I}$ and the derivative time $T_{D}$ are determined [1]. (Design of a similar controller is covered in [6]). LEDA operational experience is also discussed.

\section{PREDICTED AMPLITUDE MARGINS OF LLRF CONTROL SYSTEM}

The relay experiment is also applicable as part of the identification process of the ultimate gain $K_{c r}$ and the ultimate frequency $f_{c r}$, calculated from the stable limit cycles. Based on the measured ultimate gain and ultimate frequency, we can estimate the amplitude margin of the closed loop system. Let $G_{l}(i \omega)$ be the loop transfer function of the closed loop system. The ultimate frequency $f_{c r}$ is the frequency where the phase of the closed loop transfer function $G_{l}(i \omega)$ i $\mathrm{s}$ $\arg G_{l}\left(i 2 \pi f_{c r}\right)=-\pi$. The ultimate gain, $K_{c r}$ is the amplitude of the loop transfer function at the ultimate frequency $f_{c r}$. Then the amplitude margin of the closed loop system is given by

$$
\begin{aligned}
A_{m} & =\frac{1}{\left|G_{l}\left(i 2 \pi f_{c r}\right)\right|} \\
& =K_{c r}=\frac{4 M}{\pi a}
\end{aligned}
$$

where $\mathbf{M}$ is the amplitude of the ideal relay and $a$ is the limit cycle amplitude.

Figure 1 shows the Matlab set-up of the relay experiment for analyzing the LLRF control system.

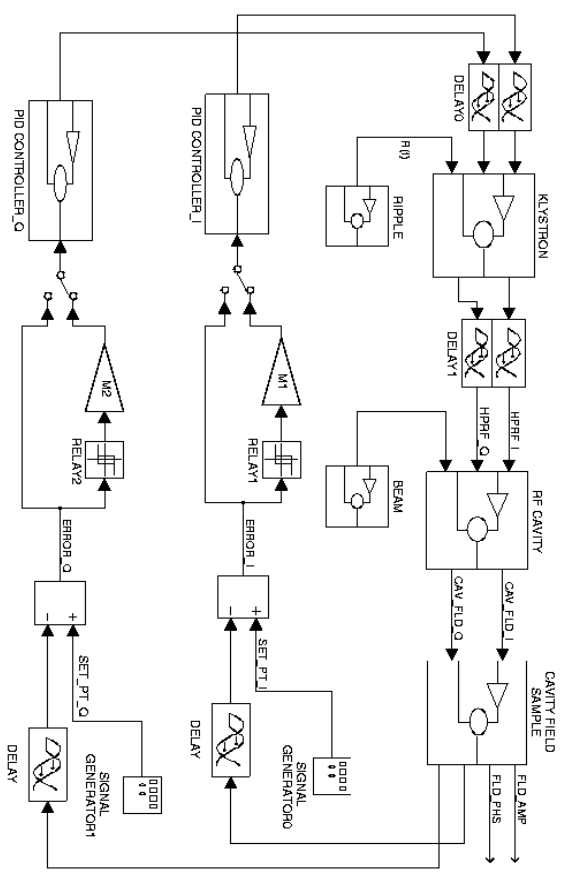

Figure 1. Relay Experiment Model.

Matlab was used to predict the amplitude margin of this model for a given square wave amplitude. Different $\mathbf{M}$ values were used for the In-phase loop, and Quadrature loop. The test results are given below for $\mathrm{M} 1=0.035$ (inphase) and M2=0.045 (quadrature).

\footnotetext{
* Work supported by the US Department of Energy.
} 
Table 1. Ultimate frequencies and amplitude margins

\begin{tabular}{|l|l|l|l|}
\hline$T_{c r}^{I}$ & $3.997 \mu \mathrm{s}$ & $T_{c r}^{Q}$ & $4.024 \mu \mathrm{s}$ \\
\hline$f_{c r I}$ & $250.2 \mathrm{kHz}$ & $f_{c r Q}$ & $248.5 \mathrm{kHz}$ \\
\hline$a I$ & 0.164 & $a Q$ & 0.029 \\
\hline$A_{m}^{I}$ & 2.712 & $A_{m}^{Q}$ & 1.967 \\
\hline
\end{tabular}

$A_{m}^{I}$ and $A_{m}^{Q}$ are the amplitude margins of the loop transfer functions $G_{l}^{I}(s)=C_{I}(s) G_{I}(s) \quad$ and $G_{l}^{Q}(s)=C_{Q}(s) G_{Q}(s)[1]$, respectively, where $G_{l}^{I}(s)$ represents the transfer function from LLRF_I to FLD_I and $G_{l}^{Q}(s)$ represents the transfer function from LLRF_Q to FLD_Q. Amplitude margins typically vary from 2.0 to $5.0[1]$.

\section{LEDA OPERATIONS}

The LLRF control system has gone through much development and system integration on the LEDA facility these past few months. In addition to providing open loop and closed loop continuous wave $(\mathrm{CW})$ in-phase and quadrature (I/Q) field control performance, it also operates in a pulsed mode. Also, cavity field amplitude-only control is included as a means of conditioning the RFQ. In order to condition the LEDA RFQ, it is necessary to operate in a controlled amplitude-modulated scenario, where a higher RF pulse amplitude on top of a lower DC level is injected into the cavity. Because the RFQ drifts in frequency with the amount of RF power in it, this control must be able to operate at frequencies other than just $350.000 \mathrm{MHz}$.

\subsection{Implementation}

As reported previously, the implementation of the LEDA LLRF control system is five LANL-designed VXIbus modules [2]. The Field Control Module has two parallel circuitry paths for control: a digital portion (centered around a digital signal processor (DSP)), and an analog portion. Operational requirements for field Amplitude Control are met through the use of the DSP portion only. We measure the in-phase and quadrature portion of the cavity field in the RFQ, calculate the square of its magnitude, and compare this to the square of the setpoints. This method eases the computational time required to perform square roots in the DSP (via a lengthy Taylor series expansion).

\subsection{Field Control}

The Field Control Module is setup to run in six independent modes as defined by the operator: CW Open Loop, CW Amplitude Control, CW I/Q Control, and their Pulsed counterparts. These are used for Calibration, Conditioning, Turn-on, and Normal Operations. The following figure indicates the differences in the cavity field for each mode, and what is being controlled.
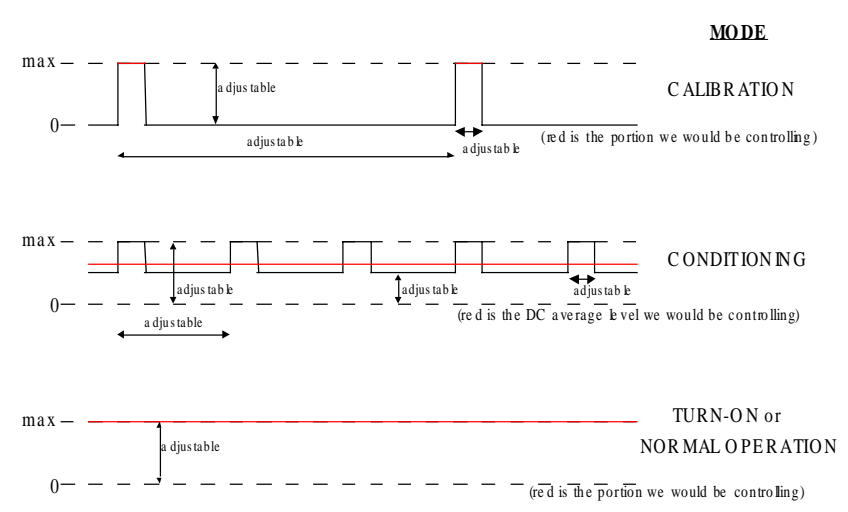

Figure 2. Modes of Operation

Field Amplitude Control is implemented in the FCM in such a way as to provide a broader system response at the expense of the bandwidth of field I/Q control, since the system must operate at whatever frequency the RFQ resonates, typically $\pm 250 \mathrm{kHz}$ around $350 \mathrm{MHz}$. These two control schemes are implemented quite differently and hence, system response differs dramatically. Initially, we intended to have many different digital decimating filter (DDF) coefficient sets to accommodate different system responses. For example, in order to achieve field control at the wide $350 \mathrm{MHz} \pm 250 \mathrm{kHz}$ frequencies, we slowed the on-board (DDF) coefficients to a set that had $250 \mathrm{kHz}$ bandwidth, $1 \mu \mathrm{s}$ data rate thereby providing amplitude control only at low bandwidth $(\sim 10 \mathrm{~Hz})$. When we implement full I/Q control, the DDF coefficient set was to have a $25 \mathrm{kHz}$ bandwidth, $16 \mu$ s data rate thereby providing full I/Q control at the full bandwidth $(\sim 10 \mathrm{kHz})$. During LEDA operations, however, we have found that the short time required to switch modes causes a brief transient in klystron drive which in turn trips reflected power monitors and hence, turns things off. Therefore we have changed this operation philosophy to performing Amplitude only, and full I/Q control both at the $25 \mu$ s data rate, such that the closed loop bandwidth will be on the order of $10-15 \mathrm{kHz}$.

\subsection{Resonance Control}

At the same time cavity field Amplitude Control is performed, the LLRF system monitors and tracks the resonance frequency of the cavity [3]. In order to raise the power into the cavity correctly without creating a lot of high reflected power faults, we have found it necessary to start at a low power level, at low duty cycle, allow the resonance control module to find the resonance frequency of the cavity (typically $350.250 \mathrm{MHz}$ ) and bring that resonance frequency in to around $350.000 \mathrm{MHz}$. The cooling water system chills the RFQ such that its resonant frequency swings from $350.350 \mathrm{MHz}$ without RF power to $349.800 \mathrm{MHz}$ with RF power. This implies that the 
frequency agile mode of the Resonance Control Module must be able to track and maintain the cavity's resonance condition throughout this range. It does so by calculating the error of the transmitted equation given below

$\operatorname{Im}\left(\mathrm{Y}_{\mathrm{C}}\right)=0.2\left[\mathrm{I}_{\mathrm{T}} \cdot \mathrm{Q}_{\mathrm{F}}-\mathrm{I}_{\mathrm{F}} \cdot \mathrm{Q}_{\mathrm{T}}\right] /\left[\left(\mathrm{I}_{\mathrm{F}}+\mathrm{I}_{\mathrm{R}}\right)^{2}+\left(\mathrm{Q}_{\mathrm{F}}+\mathrm{Q}_{\mathrm{R}}\right)^{2}\right]$

where $\mathrm{Y}_{\mathrm{C}}$ is the cavity admittance, $\mathrm{I}_{\mathrm{F}}$ and $\mathrm{Q}_{\mathrm{F}}$ are the inphase and quadrature components of the forward signal, and $\mathrm{I}_{\mathrm{T}}$ and $\mathrm{Q}_{\mathrm{T}}$ are the in- phase and quadrature components of the transmitted signal in the cavity, and keeping it zero through a proportional integral algorithm. Reference 4 discusses this thoroughly. Implementing this function on the actual RFQ has not been trivial.

Because the frequency shifting is implemented digitally, there is a discrete $90^{\circ}$ phase shift which occurs between the positive and negative side of $350.000 \mathrm{MHz}$. This phase reversal is enough to cause a high reflected power spike which momentarily trips off the klystron. In order to minimize the occurrence of this phase jump, we have implemented a programmable "deadband zone" around 350.000 MHz. Now as the module tracks the RFQ resonant frequency in towards $350.000 \mathrm{MHz}$ from the outer "RFQ off-resonance" extremes, it enters a frequency deadband where it automatically switches to the 350.000 $\mathrm{MHz}$ master oscillator and allows just the water control system [5] to keep the RFQ on resonance. Should the RFQ drift out of this region, we then automatically switch back to frequency tracking. To minimize the number of phase jumps, we have also built in some hysteresis into this deadband zone such that we switch into it at \pm 0.5 $\mathrm{kHz}$, but do not switch out of it until $\pm 8 \mathrm{kHz}$.

\subsection{Software}

The software control of the LLRF system can be performed with either LabVIEW or EPICS. Both have been implemented. A typical EPICS field control operating screen is shown below. Note that it provides displays of both the analog and digital history buffers, allowing the user to debug the performance of both sets of control circuitry. Because Amplitude control is performed with the digital portion of the Field Control Module only, the digital history buffer is the only one of real interest. The display below shows the various control parameters which identify the mode and all of its setpoints for field control.

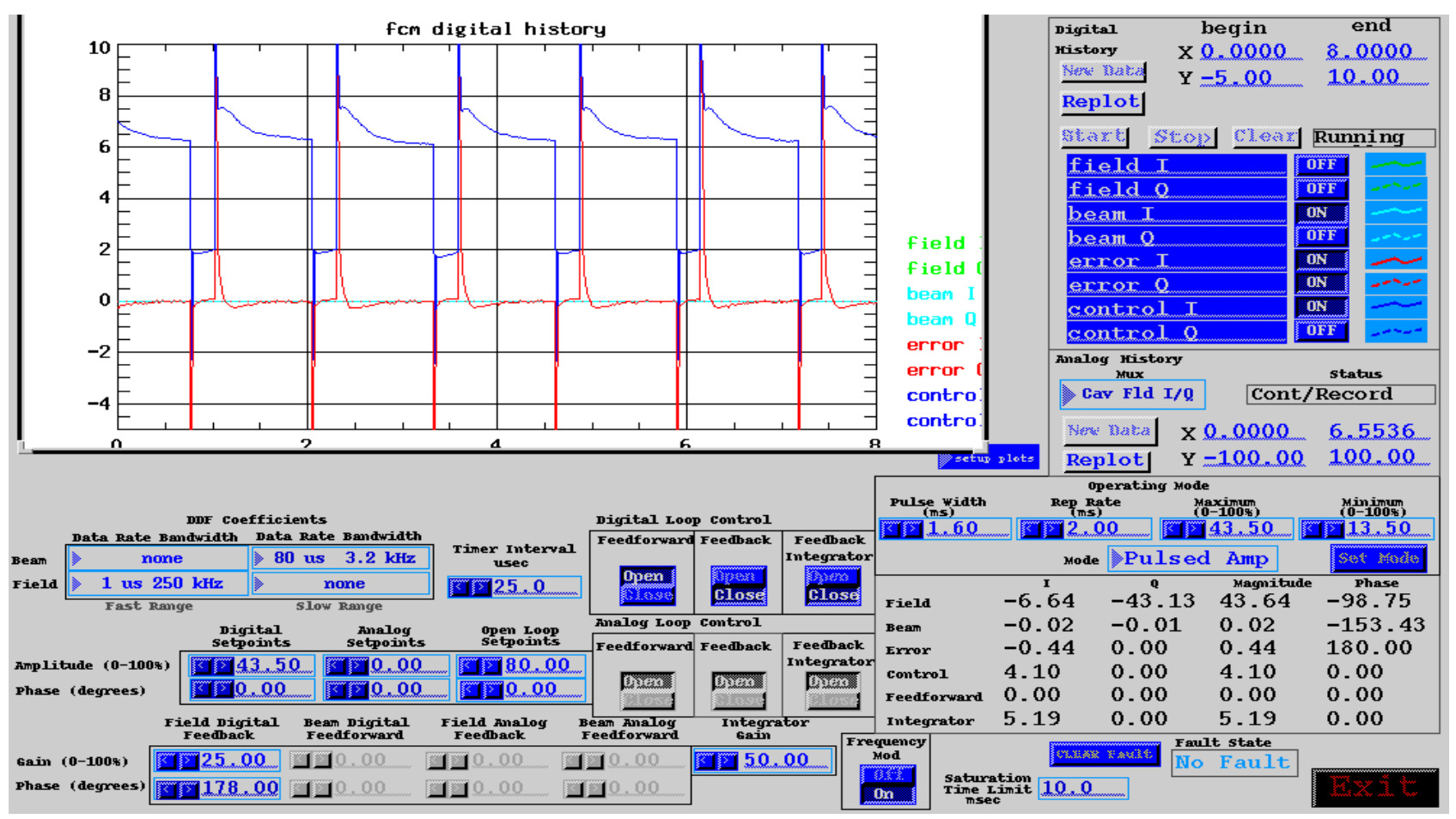

\section{REFERENCES}

1- K. J. Astrom and T. Hagglund, PID controllers: theory, design, and tuning, 2nd edn. Instrument Society of America, 1995, Research Triangle Park, NC.

2- Regan, A., et al, “APT LLRF Control System Functionality and Architecture, "Proceedings of Linear Accelerator Conference 1996, Geneva, Switzerland, August 1996, pp. 225-227.
Figure 3. Field Control display

3- Wang, Y.M., and Regan, A.H. "Algorithms and Implementations of APT Resonant Control System," PAC97.

4- Ziomek, C.D. et al, "I/Q Resonance Control Algorithms," Tech Note AOT-5-TN:008, LANL LANSCE-5 , 3/4/97.

5- Floeresch, R. et al. "Resonance Control Cooling System for the APT/ LEDA CCDTL Hot Model," MOP-1 these Proceedings.

6- Kwon, S. et al "SNS LLRF Control System model Design," These Proceedings. 